\title{
Nitric Oxide Released from Activated Platelets Inhibits Platelet Recruitment
}

\author{
Jane E. Freedman, ${ }^{\star}$ Joseph Loscalzo, ${ }^{*}$ Marc R. Barnard, ${ }^{\ddagger}$ Caroline Alpert, ${ }^{\star}$ John F. Keaney, Jr., ${ }^{\star}$ and Alan D. Michelson ${ }^{\ddagger \S}$ \\ *Whitaker Cardiovascular Institute and Evans Memorial Department of Medicine, Boston University School of Medicine, Boston, \\ Massachusetts 02118; and ${ }^{\ddagger}$ Center for Platelet Function Studies, ${ }^{\S}$ Department of Pediatrics and $\|^{D}$ Department of Surgery, University of \\ Massachusetts Medical School, Worcester, Massachusetts 01655
}

\begin{abstract}
Vessel injury and thrombus formation are the cause of most ischemic coronary syndromes and, in this setting, activated platelets stimulate platelet recruitment to the growing thrombus. Recently, a constitutive nitric oxide synthase (NOS) has been identified in human platelets. To further define the capacity of platelets to produce nitric oxide (NO), as well as to study the role of this $\mathrm{NO}$ in platelet recruitment, we adapted a NO-selective microelectrode for use in a standard platelet aggregometer, thereby permitting simultaneous measurement of platelet aggregation and NO production. Treatment of platelets with the NO synthase inhibitor $\mathrm{L}-N^{\mathrm{G}}$-nitroarginine methyl ester (L-NAME), reduced NO production by $92 \pm 8 \%$ in response to $5 \mu \mathrm{M}$ ADP compared to control but increased aggregation by only $15 \pm 2 \%$. In contrast, L-NAME had a more pronounced effect on platelet recruitment as evidenced by a $35 \pm 5 \%$ increase in the extent of aggregation, a $33 \pm 3 \%$ decrease in cyclic GMP content, and a $31 \pm 5 \%$ increase in serotonin release from a second recruitable population of platelets added to stimulated platelets at the peak of NO production. To study platelet recruitment accurately, we developed an assay that monitors two platelet populations simultaneously. Nonbiotinylated platelets were incubated with L-NAME or vehicle and activated with ADP. At peak NO production, biotinylated platelets were added. As measured by three-color flow cytometry, there was a $56 \pm 11 \%$ increase in the number of $P$ selectinpositive platelets in the nonbiotinylated population treated with L-NAME as compared to control. When biotinylated platelets were added to the L-NAME-treated nonbiotinylated population, the number of $P$ selectin positive biotinylated platelets increased by $180 \pm 32 \%$ as compared to biotinylated platelets added to the control. In summary, stimulated platelets produce NO that modestly inhibits platelet activation but markedly inhibits additional platelet recruitment. These data suggest that platelet-derived NO may regulate platelet re-
\end{abstract}

This work was presented in part at the 69th Scientific Session of the American Heart Association, 10-13 November 1996, in New Orleans, LA.

Address correspondence to Jane E. Freedman, Whitaker Cardiovascular Institute, Room W507, Boston University School of Medicine, 80 E. Concord Street, Boston, MA 02118-2394. Phone: 617-6385428; FAX: 617-638-4066.

Received for publication 3 December 1996 and accepted in revised form 11 April 1997.

J. Clin. Invest.

(C) The American Society for Clinical Investigation, Inc.

0021-9738/97/07/0350/07 \$2.00

Volume 100, Number 2, July 1997, 350-356 cruitment to a growing thrombus. (J. Clin. Invest. 1997. 100: 350-356.) Key words: nitric oxide $\bullet$ blood platelet $\bullet$ thrombosis $\bullet$ nitric oxide synthase $\cdot P$ selectin

\section{Introduction}

Thrombus formation within a coronary vessel is the acute precipitating event in most unstable ischemic coronary syndromes, as documented by angiographic (1) and pathologic (2) studies. Increased platelet-derived thromboxane (3) has been detected in the plasma of patients with acute myocardial infarction and unstable angina, providing biochemical support for platelet activation as an important element in these clinical syndromes. Angiographic severity of coronary stenosis, however, does not adequately predict the location of subsequent coronary artery occlusion (4). Therefore, rupture of atheromatous plaque and subsequent occlusive thrombus formation are believed responsible for most acute coronary syndromes $(5,6)$. Both superficial intimal injury caused by endothelial denudation or deep intimal injury caused by plaque rupture expose collagen and von Willebrand factor to platelets. This event leads to the adherence of platelets to the subendothelium and, subsequently, platelet activation. Once activated, platelets further stimulate thrombus formation by releasing ADP and serotonin, synthesizing thromboxane $\mathrm{A}_{2}$, and promoting thrombin generation at the platelet surface. This process, which activates additional platelets and recruits them to the site of vascular injury, is known as the recruitment phase of hemostasis or thrombosis (7).

Adhesion of platelets to the vessel wall is prevented, in part, by endothelial cell production of nitric oxide $(\mathrm{NO})^{1}(8,9)$. Nitric oxide also inhibits platelet aggregation $(10,11)$ and prevents thrombosis in a model of endotoxin-induced glomerular damage (12). The normal activation-dependent increase in platelet surface glycoprotein expression, including $\mathrm{P}$ selectin and the activated glycoprotein IIb-IIIa complex (integrin $\left.\alpha_{\mathrm{IIb}} \beta_{3}\right)$, is also inhibited by NO (13).

A constitutive nitric oxide synthase (cNOS) has been identified in both human platelets and megakaryoblastic cells (14). Nitric oxide is also produced within the context of platelet aggregation, and platelet-derived NO appears involved in the regulation of platelet function as aggregation is enhanced by incubation with inhibitors of NOS and inhibited by incubation with the NOS substrate, L-arginine (15). Nitric oxide release from activated human platelets has been indirectly measured and appears comparable to that of endothelial cells (16). In

1. Abbreviations used in this paper: cNOS, constitutive nitric oxide synthase; GFP, gel-filtered platelets; L-NAME, L- $N^{\mathrm{G}}$-nitroarginine methyl ester; L-NMMA, L- $N^{\mathrm{G}}$-monomethyl arginine citrate; NO, nitric oxide; PE, phycoerythrin; PRP, platelet-rich plasma; RGDS, L-Arg-L-Gly-L-Asp-L-Ser. 
vivo, systemic infusion of the NOS inhibitor $\mathrm{L}-N^{\mathrm{G}}$-monomethyl arginine citrate (L-NMMA) causes a reduction in bleeding time without a change in vessel tone (17) and enhances platelet reactivity to various agonists (18).

Under resting conditions, the source of $\mathrm{NO}$ acting on platelets is most likely derived from shear forces on the endothelium but, under conditions associated with disrupted endothelium and platelet activation, platelet-derived nitric oxide may also play a role in modulating platelet recruitment. In this study, we directly measure and characterize aggregation-induced platelet-derived NO production so as to determine its effect on primary platelet aggregation as well as to study its role in platelet recruitment.

\section{Methods}

Chemicals and solutions. L-arginine, L-cysteine, sodium nitrite, imipramine, BSA, hydrochloric acid, Sepharose 2B, Hepes, ADP, collagen, thrombin, U46619, PMA, L-Arg-L-Gly-L-Asp-L-Ser (RGDS), and GSH were purchased from Sigma Chemical Co. (St. Louis, MO). Tyrode's Hepes-buffered saline consisted of $140 \mathrm{mM} \mathrm{NaCl}, 6 \mathrm{mM}$ Hepes, $2 \mathrm{mM} \mathrm{Na}_{2} \mathrm{HPO}_{4}, 2 \mathrm{mM} \mathrm{MgSO}$, $0.1 \%$ dextrose, $\mathrm{pH} 7.4$, and $0.4 \%$ BSA. L- $N^{\mathrm{G}}$-nitroarginine methyl ester (L-NAME), L-NMMA, diethylamine NONOate, U46619, and cyclic GMP enzyme immunoassay kits were purchased from Cayman Chemical Co. (Ann Arbor, MI). $\left[{ }^{14} \mathrm{C}\right]$ serotonin was purchased from Amersham Corp. (Arlington Heights, IL). Biotin $N$-hydroxysuccinimide ester (NHS-biotin) was purchased from Calbiochem (San Diego, CA).

Monoclonal antibodies. PB1.3 (Centocor, Malvern, PA) is a murine monoclonal antibody directed against $\mathrm{P}$ selectin (19). PB1.3 and murine $\mathrm{IgG}_{1}$ were conjugated with FITC using a Quick Tag Kit (Boehringer Mannheim Corp., Indianapolis, IN). Phycoerythrin (PE) conjugated anti-CD41 monoclonal antibody was purchased from Dako Corp. (Carpinteria, CA).

Preparation of platelet-rich plasma and gel-filtered platelets. Peripheral blood was drawn using standard methodology from healthy adult volunteers who had not consumed acetylsalicylic acid or any other platelet inhibitor for at least $10 \mathrm{~d}$ (20). The citrated blood was centrifuged $\left(150 \mathrm{~g}, 15 \mathrm{~min}, 22^{\circ} \mathrm{C}\right)$ and the supernatant, representing platelet-rich plasma (PRP), was separated. Gel-filtered platelets (GFP) were prepared by passing PRP over a Sepharose-2B column equilibrated with Tyrode's Hepes-buffered saline, as previously described (21). Platelet counts were determined using a Coulter counter (ZM; Coulter Electronics, Miami, FL).

Platelet aggregation. Aggregation of GFP was monitored using a standard nephelometric technique (22). Platelet aggregation experiments were conducted in the presence of $2 \mathrm{mM} \mathrm{CaCl}_{2}, 90 \mu \mathrm{M} \mathrm{MgCl}_{2}$, and $1 \mathrm{mg} / \mathrm{ml}$ fibrinogen in Tyrode's Hepes-buffered saline unless otherwise indicated. Agonist-induced aggregation was recorded as the absolute extent of change in light transmittance using a four-chamber aggregometer (BioData, Hatboro, PA).

Measurement of platelet nitric oxide production and aggregation. We adapted a NO-selective (23) microelectrode (Inter Medical Co., Ltd., Nagoya, Japan) for use in a standard platelet aggregometer (Payton Associates, Buffalo, NY) to monitor platelet NO production and aggregation simultaneously. The NO monitoring device consists of a headstage amplifier with a built-in power supply and two electrodes. The working electrode $(0.2 \mathrm{~mm}$ diameter, made from $\mathrm{Pt} / \mathrm{Ir}$ alloy) and counterelectrode were inserted in an aggregometer microcuvette containing a stir bar using a micromanipulator. The aggregometer, electrode, and headstage amplifier were housed in a Faraday cage to reduce electrical interference. The electrode current increased linearly from 10 to $350 \mathrm{pA}$ over a range of NO concentrations. All experiments were conducted at constant temperature $\left(37^{\circ} \mathrm{C}\right)$. Production of $\mathrm{NO}$ was determined by integrating the area under the response curve. Diethylamine NONOate was used to derive a standard curve which was linear over the range of $10-500 \mathrm{nM}$, the correlation coefficient for which was typically 0.95 .

Over the range of $0-5 \times 10^{8}$ activated platelets we observed a dosedependent increase in platelet NO production with a correlation coefficient of 0.93 . There was also a dose-dependent increase in NO production with respect to ADP concentration from 0-2 $\mu \mathrm{M}$ using a fixed number of platelets. To assess interassay reproducibility, GFP prepared from 10 normal controls were studied on two different days. For each donor, the average difference in between day determinations of NO production was $19 \pm 10 \%$. The average within day difference between determinations of NO production was $14 \pm 7 \%$ for each donor.

Cyclic nucleotide assay. Trichloroacetic acid (final concentration, $5 \% \mathrm{vol} / \mathrm{vol}$ ) was added to GFP. Samples were vortexed, placed on ice, and centrifuged at $1,500 \mathrm{~g}$ for $10 \mathrm{~min}$ at $4^{\circ} \mathrm{C}$. The supernatant was extracted with diethyl ether and assayed for cGMP by an ELISA using cGMP antiserum (Cayman Chemical Co.).

Platelet secretion. Platelet secretion was measured using $\left[{ }^{14} \mathrm{C}\right]-$ radiolabeled serotonin as previously described $(9,24)$. GFP were incubated with $\left[{ }^{14} \mathrm{C}\right]$ serotonin at $37^{\circ} \mathrm{C}$ for $10 \mathrm{~min}$. Imipramine was added immediately before the initiation of secretion to prevent reuptake of secreted serotonin. Secretion was initiated by the addition of $5 \mu \mathrm{M}$ ADP, allowed to proceed for $2 \mathrm{~min}$, and then terminated by the addition of ice-cold formaldehyde in 0.05 M EDTA. Samples were spun in a microfuge at $14,000 \mathrm{~g}$ for $3 \mathrm{~min}$ and radioactivity was measured in the supernatant.

Preparation of platelets for flow cytometry. PRP was incubated for $15 \mathrm{~min}$ at $22^{\circ} \mathrm{C}$ with or without $200 \mu \mathrm{g} / \mathrm{ml}$ NHS-biotin followed by gel-filtration. Platelets were then treated with either $300 \mu \mathrm{M}$ L-NAME, $1 \mathrm{mM}$ L-arginine, or vehicle for $15 \mathrm{~min}$ at $22^{\circ} \mathrm{C}$. Nonbiotinylated GFP were then mixed in an aggregometer at $37^{\circ} \mathrm{C}$ with $200 \mu \mathrm{M}$ RGDS and $2 \mu \mathrm{M}$ ADP to induce platelet activation and platelet-derived NO production in the absence of platelet aggregation. The inability to aggregate was confirmed by aggregometry as well as by flow cytometry. At the peak of NO production, biotinylated GFP were added. After $2 \mathrm{~min}$, additional agonist was added ( $20 \mu \mathrm{M}$ ADP) followed by an additional 3-min incubation $\left(37^{\circ} \mathrm{C}\right)$. Samples were then fixed in formaldehyde ( $1 \%$ final concentration) for $20 \mathrm{~min}$ at $22^{\circ} \mathrm{C}$ and diluted 10 -fold in modified Hepes Tyrode's buffer $(137 \mathrm{mM} \mathrm{NaCl}, 2.8 \mathrm{mM} \mathrm{KCl}, 1 \mathrm{mM}$ $\mathrm{MgCl}_{2}, 12 \mathrm{mM} \mathrm{NaHCO}, 0.4 \mathrm{mM} \mathrm{Na}_{2} \mathrm{HPO}_{4}, 0.35 \%$ bovine serum albumin, $10 \mathrm{mM}$ Hepes, $5.5 \mathrm{mM}$ glucose, $\mathrm{pH}$ 7.4). The samples were then incubated $\left(22^{\circ} \mathrm{C}, 30 \mathrm{~min}\right)$ with a saturating concentration of FITC-conjugated monoclonal antibody PB1.3 (P selectin specific) or murine $\mathrm{IgG}_{1}$ and a near-saturating concentration of phycoerythrinconjugated anti-CD41 monoclonal antibody and streptavidin-RED670 (Cy5-PE tandem conjugate; Gibco BRL, Grand Island, NY). All samples were next diluted 20 -fold in modified Tyrode's buffer, $\mathrm{pH}$ 7.4, and analyzed immediately.

Flow cytometry. Samples of GFP were analyzed in a Coulter Epics XL flow cytometer (Coulter Cytometry, Miami, FL). The flow cytometer was equipped with a $500 \mathrm{~mW}$ argon laser (Cyonics, San Jose, CA) operated at $15 \mathrm{~mW}$ and a wavelength of $488 \mathrm{~nm}$. The data were collected using 525, 575, and $675 \mathrm{~nm}$ bandpass filters for FITC, PE, and RED670, respectively. Appropriate color compensation settings were established. Platelets were distinguished by their characteristic light scatter (log scale) and binding of anti-CD41-PE monoclonal antibody (discriminator set on the CD41 parameter). Based on their RED670 signal, biotin-positive and negative platelets were differentiated in the same sample. Data were collected for 12,000 platelets/ sample. The percentage of cells positive for $\mathrm{P}$ selectin was generated by subtracting the isotype control (mouse $\mathrm{IgG}_{1}-\mathrm{FITC}$ ) from the PB1.3-FITC test using IMMUNO-4 histogram processing with "Oversub On" (Coulter Epics XL software, Coulter cytometry).

Statistics. All data are presented as the mean \pm standard error of the mean. Paired samples were compared by Student's $t$ test; values of $P<0.05$ were considered significant. Groups of data were tested by analysis of variance and, if significant, were further evaluated by the Newman-Keuls test. 


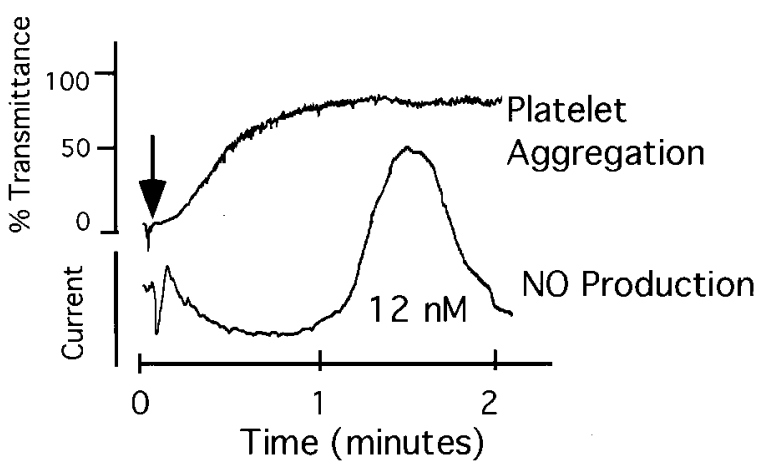

Figure 1. Effect of platelet stimulation on platelet NO production. Representative tracing of gel-filtered platelets stimulated with ADP followed by simultaneous measurement of platelet aggregation (percent transmittance) and NO production (corresponding to $12 \mathrm{nM}$ diethylamine NONOate peak) as described in Methods. Arrow indicates the time of addition of ADP. Tracing is representative of five experiments.

\section{Results}

Characterization of nitric oxide production and oxygen consumption in activated platelets

To study the relationship between NO production and platelet aggregation, platelets were stimulated with $5 \mu \mathrm{M}$ ADP and $\mathrm{NO}$ measured as described in Methods. NO production began $\sim 1.2 \mathrm{~min}$ after addition of ADP and peaked at $1.6 \mathrm{~min}$ (Fig. 1). In contrast, the addition of diethylamine NONOate or $\mathrm{S}$-nitroso-L-cysteine to unstimulated GFP produced a peak within $5 \mathrm{~s}$ (data not shown). To confirm the signal as platelet NO production, PRP was incubated with $300 \mu \mathrm{M}$ L-NAME or $1 \mathrm{mM} \mathrm{L}$-arginine for $15 \mathrm{~min}$ at $37^{\circ} \mathrm{C}$ and then, after gel-filtration, the platelets were stimulated by the addition of $5 \mu \mathrm{M}$ ADP. Incubation with the NOS inhibitor, L-NAME, reduced aggregation-dependent NO production by $92 \pm 8 \%(n=5)$ while incubation with the NOS substrate L-arginine increased NO production by $50 \pm 17 \%(n=5)$ compared with control platelets (Table I). Decreased platelet-derived NO production was associated with enhancement of aggregation.

To determine the effect of other platelet agonists on platelet-derived NO production, GFP were stimulated with collagen, thrombin, the thromboxane $\mathrm{A}_{2}$ analog U46619, or PMA. Thrombin and U46619 induced NO production but to a lesser extent than did ADP (data not shown). PMA induced a large nonspecific signal and collagen resulted in platelet adherence to the microelectrode and distortion of the signal. Because of these findings, all subsequent aggregation experiments were carried out with ADP as agonist. In addition, stimulation of platelets from donors who had taken aspirin $(325 \mathrm{mg})$ with 5 $\mu \mathrm{M}$ ADP did not alter platelet NO production.

\section{Effect of platelet-derived nitric oxide on platelet recruitment}

Aggregation. As shown in Table I, inhibition of platelet NOS only modestly altered platelet aggregation. In addition, the peak NO signal produced by activated platelets began more than one minute after ADP stimulation (Fig. 1). The measurement of peak NO release after the completion of aggregation suggested that, in addition to influencing the primary platelet aggregatory response, platelet release of nitric oxide may also be important for the attenuation of platelet recruitment. To in-
Table I. Effect of L-NAME and L-Arginine on Platelet NO Production and Aggregation Response

\begin{tabular}{lcc}
\hline & NO & $\begin{array}{c}\text { Extent of } \\
\text { aggregation }\end{array}$ \\
\hline & pmol/10 $0^{8}$ platelets & $\%$ \\
Platelets & 0.0 & 0 \\
Platelets + ADP & $4.8 \pm 1.2$ & $64 \pm 5$ \\
Platelets + L-NAME + ADP & $0.4 \pm 0.2^{*}$ & $74 \pm 4^{*}$ \\
Platelets + L-arginine + ADP & $7.2 \pm 1.5^{*}$ & $59 \pm 11$ \\
\end{tabular}

Platelets in Hepes buffer were incubated with L-arginine, L-NAME, or vehicle followed by activation with ADP and measurement of NO production and aggregation response. ${ }^{*} P<0.05$ vs control platelets; expressed as mean \pm SEM for five experiments using equivalent numbers of platelets in each experiment.

vestigate the role of platelet-derived $\mathrm{NO}$ in platelet recruitment, GFP that had been incubated with $300 \mu \mathrm{M}$ L-NAME, $1 \mathrm{mM}$ L-arginine, or vehicle control, were stimulated with ADP and, during peak NO production, additional untreated GFP were added (Fig. 2). Platelets added to GFP preincubated with L-NAME, which produced minimal NO, demonstrated increased aggregation. Addition of platelets to GFP preincubated with $1 \mathrm{mM}$ L-arginine did not lead to significant differences in aggregation as compared to control (Fig. 2). Addition of GFP to Tyrode's Hepes buffer containing L-NAME, L-arginine, or control in the presence of ADP did not lead to any change in platelet aggregation. Addition of L-NAME to control platelets at the time of platelet stimulation with ADP also did not alter the extent of aggregation of the subsequently added GFP as compared with the addition of control platelets alone.

Previous studies of platelet recruitment have involved the isolation of the supernatant from activated platelets and mixed the supernatant with untreated platelets (7). Therefore, GFP were incubated with $300 \mu \mathrm{M}$ L-NAME, $1 \mathrm{mM}$ L-arginine, or vehicle control, and stimulated with ADP. Just before peak NO production, the samples were microfuged at $1,400 g$ for $60 \mathrm{~s}$. The supernatant was removed and immediately added to untreated GFP. The experiment only minimally altered aggregation possibly due to the short half-life of NO. Therefore, $10 \mu \mathrm{M}$ L-cysteine was added to the GFP allowing for nitrosation and

Table II. The Effect of Platelet-derived NO on cGMP Production and Secretion by Recruited Platelets

\begin{tabular}{lc}
\hline & cGMP \\
\hline & pmol/10 $0^{8}$ platelets \\
Stimulated GFP & $1.6 \pm 0.2$ \\
GFP added to untreated platelets & $1.8 \pm 0.3$ \\
GFP added to L-arginine-treated platelets & $2.1 \pm 0.3$ \\
GFP added to L-NAME-treated platelets & $1.2 \pm 0.1^{*}$
\end{tabular}

Platelets were incubated with L-arginine, L-NAME, or vehicle and stimulated with $5 \mu \mathrm{M}$ ADP. During peak NO production, untreated GFP were added and measured for platelet cGMP levels. cGMP content is expressed as the mean \pm SEM for three experiments. $* P<0.05$ vs untreated platelets. 


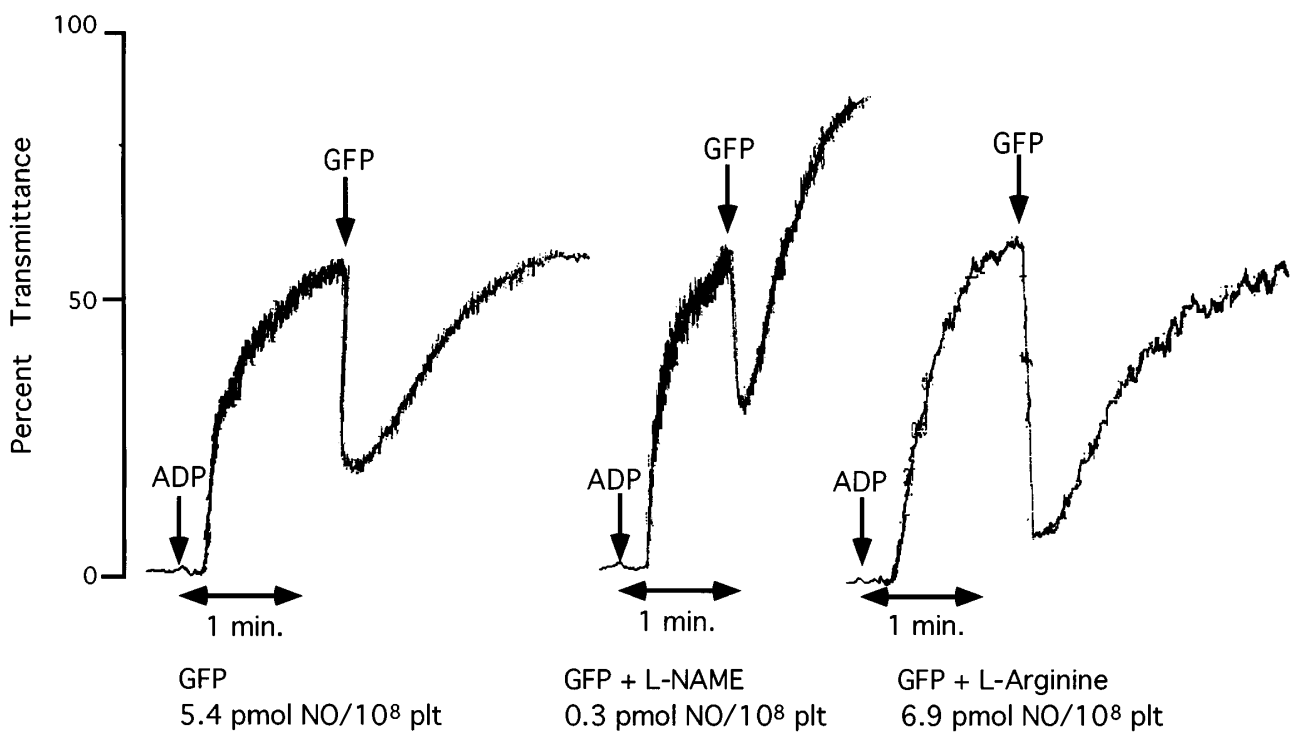

Figure 2. Representative tracing of the effect of plateletderived NO on subsequent platelet aggregation of a second population of recruited platelets. Gel-filtered platelets incubated with $300 \mu \mathrm{M}$ L-NAME, $1 \mathrm{mM}$ L-arginine, or vehicle were stimulated with ADP and, during peak NO production, additional untreated GFP were added. Nitric oxide production for this experiment is expressed as pmol $\mathrm{NO} / 10^{8}$ platelet and is representative of five experiments. formation of an S-nitrosothiol species with a prolonged halflife $(10,11)$. Supernatant from L-NAME treated platelets led to an increase $(91.4 \pm 8.2 \%)$ and incubation with L-arginine led to a decrease $(58.2 \pm 7.4)$ in platelet aggregation response as compared to control platelets $(63.0 \pm 8.2)$.

Nitric oxide mediates its effects by elevating intracellular levels of cGMP (25). To determine if the effect of NO on platelet recruitment is cGMP-dependent, GFP that had been incubated with L-NAME, L-arginine, or vehicle control were stimulated with ADP. At the peak of NO production, additional untreated GFP were added and cGMP measured (Table II). Consistent with the previous aggregation experiments, preincubation of platelets with L-NAME led to a decrease in platelet cGMP.

Dense granule secretion. Interpretation of the previous aggregation experiments is limited because the two platelet populations were not evaluated separately. To avoid this problem we used $\left[{ }^{14} \mathrm{C}\right]$ serotonin release, a measure of platelet dense granule secretion, as an index of platelet activation. Gel-filtered platelets incubated in the presence of $1 \mathrm{mM} \mathrm{L}$-arginine, $300 \mu \mathrm{M}$ L-NAME, or vehicle control, then stimulated with $\mathrm{ADP}$ and, at the peak of NO production, unstimulated $\left[{ }^{14} \mathrm{C}\right]-$ loaded GFP were added and serotonin release determined. When $\left[{ }^{14} \mathrm{C}\right]$ serotonin-containing platelets were added to GFP preincubated with L-NAME, there was a $31 \pm 5 \%$ increase in serotonin release. In contrast, when GFP were added to GFP preincubated with L-arginine, there was a $19 \pm 3 \%$ decrease in serotonin release as compared to control (Fig. 3; $P<0.05$ for both L-NAME and L-arginine treated platelets as compared to control). These data suggest that platelet-derived NO release inhibits dense granule secretion by recruited platelets.

Alpha granule secretion. While the previous experiment examined the effect of platelet-derived NO on activation of a recruitable platelet population, it does not allow for simultaneous assessment of the NO donor platelet population. To overcome this limitation, we used a double-labeling technique. One population of platelets were biotinylated to allow for separation of the NO-donor and recruited platelet populations by flow cytometry. A representative scatter histogram demon- strating separation of the $\mathrm{NO}$ releasing and recruitable platelet populations based on biotinylation is shown in Fig. $4 A$.

Using this technique, the effect of platelet-derived $\mathrm{NO}$ on platelet recruitment was assessed by measuring surface expression of $P$ selectin, which reflects $\alpha$ granule secretion. Nonbiotinylated GFP, which had been preincubated with L-NAME, L-arginine, or vehicle, were stimulated with $2 \mu \mathrm{M}$ ADP and, during peak NO production, biotinylated (recruitable) GFP were added. After a 3-min incubation and restimulation with $20 \mu \mathrm{M}$ ADP, samples were fixed and analyzed by flow cytometry with the $\mathrm{P}$ selectin-specific monoclonal antibody PB1.3.

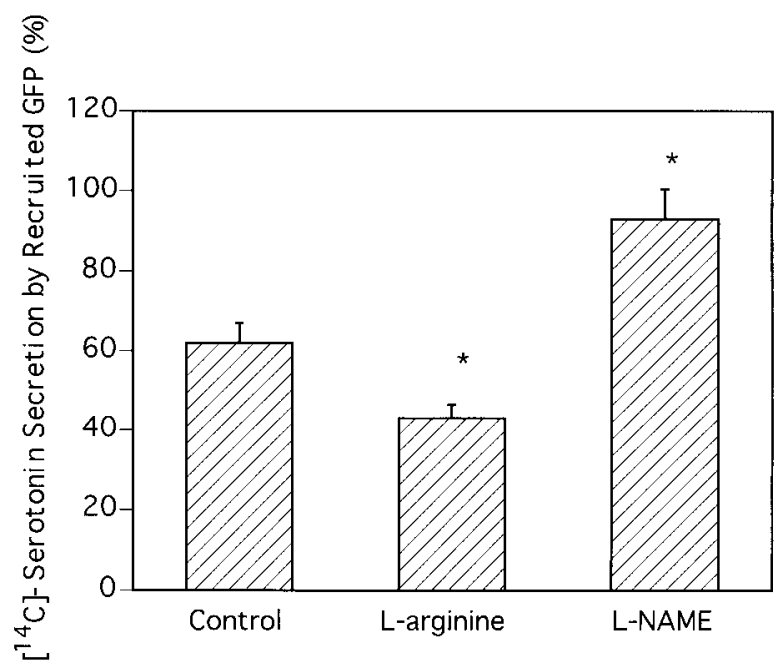

Figure 3. Effect of platelet-derived NO production on platelet recruitment as determined by measurement of platelet dense granule secretion. Platelets were incubated with L-arginine, L-NAME, or vehicle, then activated with ADP. During estimated time of peak NO production, unstimulated GFP preincubated with $\left[{ }^{14} \mathrm{C}\right]$ serotonin were added and platelet secretion determined by measuring the release of $\left[{ }^{14} \mathrm{C}\right]$ serotonin. Serotonin secretion is expressed as the mean \pm SEM for three experiments. $* P<0.05$ vs untreated platelets. 

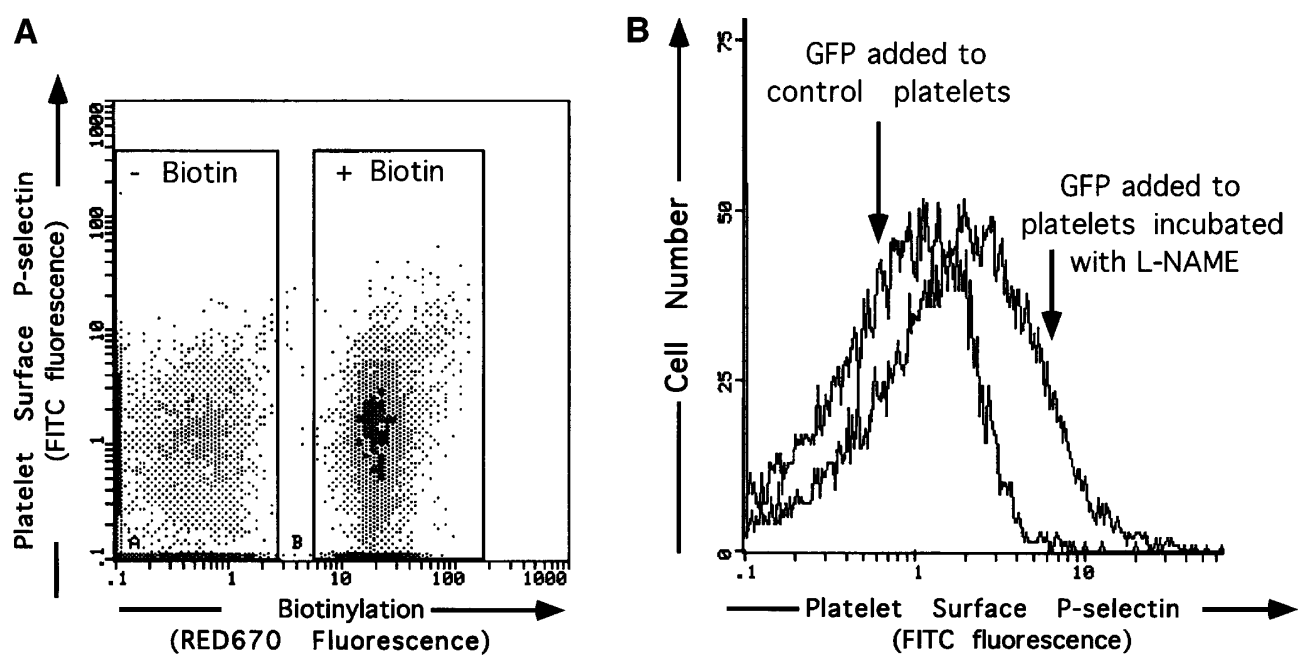

Figure 4. (A) Flow cytometric method for the simultaneous analysis of primary and recruitable platelet populations. A representative scatter histogram (of $n=4$ experiments) demonstrating separation of the primary population and added recruitable platelet population based on biotinylation ( $X$-axis) and platelet surface expression of $\mathrm{P}$ selectin ( $Y$-axis). (B) Representative histogram (of 4 experiments) demonstrating increased fluorescence (reflecting increased surface $P$ selectin expression) in GFP added to platelets incubated with L-NAME as compared with GFP added to control platelets.
For the NO donor platelets treated with L-NAME, there was a $56 \pm 11 \%$ increase in the number of platelets positive for surface $\mathrm{P}$ selectin expression as compared to control (Fig. 5). Next, $\mathrm{P}$ selectin expression in the recruitable platelet population was determined. A representative histogram demonstrates increased platelet surface $P$ selectin for GFP added to platelets incubated with L-NAME as compared with GFP

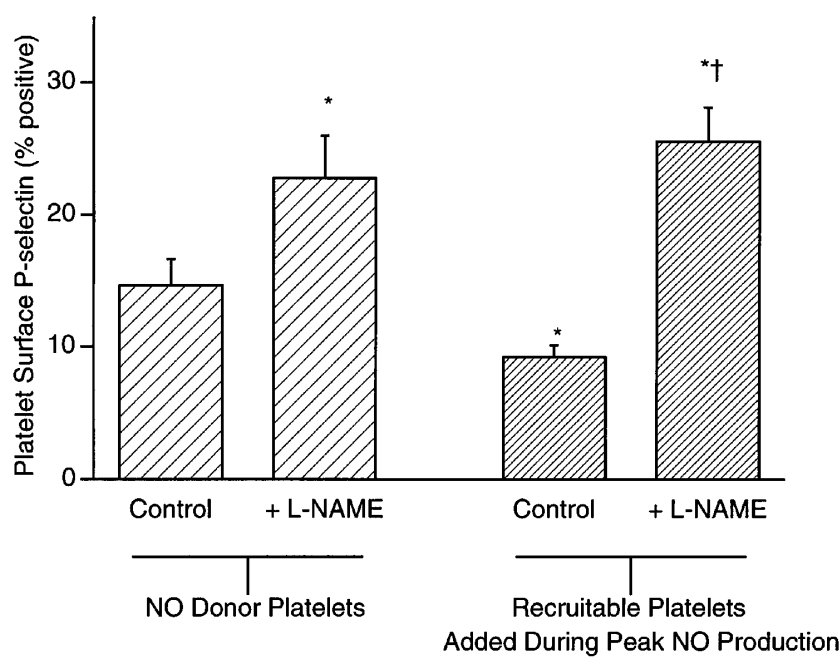

Figure 5. Effect of platelet-derived NO production on platelet recruitment, as determined by measurement of platelet alpha-granule secretion. As compared to GFP added to control platelets, inhibition of NO production by platelets incubated with L-NAME markedly increases the activation-dependent expression of platelet surface $\mathrm{P}$ selectin on recruitable GFP added during the estimated time of peak NO production. Platelets that had been incubated with L-NAME or vehicle control were stimulated with ADP and, during the estimated time of peak NO production, additional untreated GFP were added. After restimulation with ADP, samples were fixed and analyzed by flow cytometry with monoclonal antibody PB1.3 (P selectin specific). Only one of the two platelet populations were biotinylated allowing for the separate analysis. Platelet surface P selectin expression is expressed as the mean \pm SEM for four experiments. $* P<0.05$ vs control NO donor platelets; ${ }^{\dagger} P<0.01$ vs recruitable control platelets added to untreated donor platelets. added to control platelets (Fig. $4 \mathrm{~B}$ ). For GFP added to stimulated L-NAME-treated platelets, there was a $180 \pm 32 \%$ increase in surface $\mathrm{P}$ selectin expression as compared with recruitable GFP added to untreated platelets (Fig. 5). For platelets treated with L-arginine, there was a $10 \pm 2 \%$ decrease in the number of platelets positive for surface $\mathrm{P}$ selectin expression as compared to control (for $n=4$ experiments, $P=\mathrm{NS}$ ). There was a $17 \pm 4 \%$ decrease in surface $\mathrm{P}$ selectin expression for GFP added to L-arginine-treated platelets as compared with GFP added to untreated platelets (for $n=4$ experiments, $P=$ NS). At baseline, GFP were not significantly activated, as determined by the lack of platelet surface $P$ selectin expression on unstimulated samples. The experimental results were similar irrespective of whether the donor platelets or the recruitable platelets were biotinylated. These experiments show that, consistent with the previous results, incubation with L-NAME modestly increases platelet activation. More importantly, these experiments demonstrate that inhibition of NO production in platelets markedly increases the activationdependent surface expression of $\mathrm{P}$ selectin on a recruitable platelet population.

\section{Discussion}

In this study, we characterized platelet-derived NO by measuring its production simultaneously with platelet aggregation and using this information, demonstrated that platelet-derived NO is involved in the recruitment process. Previous studies have shown modest effects of platelet-derived NO on platelet aggregation but have been limited in their conclusions by the lack of real time measurements with concurrent aggregation tracings. The ability to identify the timing and extent of the NO peak produced by stimulated platelets suggests that the effects of platelet-derived NO may be more consistent with paracrine regulation of platelet function. This hypothesis is consistent with the finding of increased surface $\mathrm{P}$ selectin expression in a platelet population exposed to decreased platelet-derived NO. The development of a method which simultaneously characterizes two platelet populations has allowed us to study the effects of platelet-derived $\mathrm{NO}$ on platelet recruitment, as well as on platelet aggregation. 
Endothelial cell NO production modulates platelet function (9) and may also affect the process of recruitment. In a recent study, oral L-arginine supplementation in healthy adult males inhibited platelet aggregation, and this effect could be reversed by ex vivo incubation with L-NMMA (26). In that study (26), no change was observed in endothelium-dependent dilation of the brachial artery suggesting that oral L-arginine causes platelet-specific increases in nitric oxide production to account for the inhibition of aggregation.

Our direct measurement of NO production by activated platelets is consistent with previous studies addressing the effect of L-arginine and NOS inhibitors on platelet aggregation $(18,27)$. As determined by the microelectrode used in this study, an activated platelet can produce up to $5 \times 10^{-17} \mathrm{~mol}$ NO. Variable production of NO by platelets stimulated with different agonists may be due to differences in $\mathrm{Ca}^{2+}$ mobilization as platelet $\mathrm{NO}$ production is $\mathrm{Ca}^{2+}$ dependent (28). Another explanation may lie in the inherent interindividual variation found among blood donors.

While previous studies of platelet recruitment have evaluated the effect of platelet-derived substances that enhance aggregation (9) or neutrophil-induced inhibition of activation (29), this represents the first study of a platelet inhibitor on platelet recruitment. Regulation of NO production by cNOS may be important for platelet aggregation, but, as suggested by the delay in peak production, it may also play a role in platelet recruitment. Our results demonstrate that inhibition of platelet-derived NO production increases platelet aggregation and secretion in a recruitable platelet population (Figs. 2 and 3). These experiments are limited, however, as they do not allow for simultaneous assessment of the NO donor and recruitable platelet populations. Previous methods assessing platelet recruitment (7) are not as useful for the evaluation of short-lived species such as NO. Therefore, we developed a novel method for studying platelet recruitment which allows simultaneous evaluation of the primary and recruitable platelet populations. By using biotinylation and flow cytometry, the relative importance of platelet-derived NO production for activation of the primary platelet population as compared to its role in platelet recruitment could be simultaneously determined in these samples (Fig. 4). In the NO donor platelet population, the L-NAME-incubated platelets had increased surface P selectin expression as compared to control (Fig. 5). More importantly, in the recruitable platelet population exposed to decreased NO production, surface $\mathrm{P}$ selectin expression was markedly increased as compared with platelets exposed to standard amounts of platelet-derived NO. This experiment clearly shows that intact endogenous NO production by platelets can alter activation of subsequently recruited platelets.

Our in vitro observations are also supported by a study which shows that L-NMMA induces platelet aggregation leading to cyclic flow variations (30). That impaired platelet NO production subsequently caused increased surface platelet $\mathrm{P}$ selectin expression is also of pathophysiological relevance. Enhanced P selectin expression increases platelet adhesion to monocytes and induces the expression of tissue factor, an initiator of coagulation (31). Consistent with this observation, inhibition of platelet surface $\mathrm{P}$ selectin expression attenuates leukocyte accumulation and subsequent fibrin deposition in vivo (32).

In summary, we have characterized platelet-derived NO by measuring its production simultaneously with platelet aggrega- tion and, using this information, demonstrated that plateletderived NO is important in the regulation of platelet recruitment. These data suggest a novel mechanism by which platelet products can modulate hemostasis. Effective release of NO from endothelial cells is impaired in patients with coronary artery disease (33) and, as we have recently shown (34), NO release from platelets is also decreased in patients with coronary atherosclerosis. Because most ischemic coronary syndromes are caused by vessel injury and thrombus formation, further study is warranted to determine if altered production of NO by activated platelets contributes to the development of acute coronary events.

\section{Acknowledgments}

The authors thank Ms. Stephanie Tribuna for her expert secretarial assistance and Ms. Anne Marie Ward and Ms. Dorinda George for their technical assistance.

Dr. Freedman is the recipient of a Grant-in-Aid from the Massachusetts Affiliate of the American Heart Association and CIDA HL03556. John F. Keaney is the recipient of CIDA HL03195. This work was supported in part by National Institutes of Health grants HL48743, HL53919, and P50HL55993; a Veterans Affairs Merit Review; and a grant from NitroMed, Inc.

\section{References}

1. DeWood, M.A., J. Spores, R. Notske, L.T. Mouser, R. Burroughs, M.S. Golden, and H.T. Lang. 1980. Prevalence of total coronary occlusion during the early hours of transmural myocardial infarction. N. Engl. J. Med. 303:897-902.

2. Falk, E. 1985. Unstable angina with fatal outcome: dynamic coronary thrombosis leading to infarction and/or sudden dealth. Circulation. 71:699-708.

3. Fitzgerald, D.J., L. Roy, F. Catella, and G.A. Fitzgerald. 1986. Platelet activation in unstable coronary disease. N. Engl. J. Med. 315:983-989.

4. Little, W.C., M. Constantinescu, R.J. Applegate, M.A. Kutcher, M.T. Burrows, F.R. Kahl, and W.P. Santamore. 1988. Can coronary angiography predict the site of a subsequent myocardial infaction in patients with mild-to-moderate coronary artery disease? Circulation. 78:1157-1166.

5. Falk, E. 1983. Plaque rupture with severe pre-existing stenosis precipitating coronary thrombosis. Br. Hrt. J. 127-134.

6. Davies, M.J., and A.C. Thomas. 1984. Thrombosis and acute coronaryartery lesions in sudden cardiac ischemic death. N. Engl. J. Med. 310:1137-1140.

7. Santos, M.T., J. Valles, A.J. Marcus, L.B. Safier, J. Broekman, N. Islam, H.L. Ullman, A.M. Eiroa, and J. Aznar. 1991. Enhancement of platelet reactivity and modulation of eicosanoid production by intact erythrocytes. A new approach to platelet activation and recruitment. J. Clin. Invest. 87:571-580.

8. de Graaf, J.C., J.D. Banga, S. Moncada, R.M.J. Palmer, P.G. de Groot, and J.J. Sixma. 1992. Nitric oxide functions as an inhibitor of platelet adhesion under flow conditions. Circulation. 85:2284-2290.

9. Broekman, M.J., A.M. Eiroa, and A.J. Marcus. 1991. Inhibition of human platelet reactivity by endothelium-derived relaxing factor from human umbilical vein endothelial cells in suspension: blockade of aggregation and secretion by an aspirin-insensitive mechanism. Blood. 78:1033-1040.

10. Stamler, J., M.E. Mendelsohn, P. Amarante, D. Smick, N. Andon, P.F. Davies, J.P. Cooke, and J. Loscalzo. 1989. N-Acetylcysteine potentiates platelet inhibition by endothelium-derived relaxing factor. Circ. Res. 65:789-795.

11. Cooke, J.P., J. Stamler, N. Andon, P.F. Davies, G. McKinley, and J. Loscalzo. 1990. Flow stimulates endothelial cells to release a nitrovasodilator that is potentiated by reduced thiol. Am. J. Physiol. 259:H804-H812.

12. Shultz, P.J., and L. Raij. 1992. Endogenously synthesized nitric oxide prevents endotoxin-induced glomerular thrombosis. J. Clin. Invest. 90:17181725 .

13. Michelson, A.D., S.E. Benoit, M.I. Furman, W.L. Breckwoldt, M.J. Rohrer, M.R. Barnard, and J. Loscalzo. 1996. Effects of endothelium-derived relaxing factor/nitric oxide on platelet surface glycoproteins. Am. J. Physiol. 270:H1640H1648.

14. Sase, K., and T. Michel. 1995. Expression of constitutive endothelial nitric oxide synthase in human blood platelets. Life Sci. 57:2049-2055.

15. Chen, L.Y., and J.L. Mehta. 1996. Variable effects of L-arginine analogs on L-arginine-nitric oxide pathway in human neutrophils and platelets may relate to different nitric oxide synthase isoforms. J. Pharm. Exp. Ther. 276:253-257.

16. Zhou, Q., G.R. Hellermann, and L.P. Solomonson. 1995. Nitric oxide release from resting human platelets. Thromb. Res. 77:87-96.

17. Simon, D.I., J.S. Stamler, E. Loh, J. Loscalzo, S.A. Frances, and M.A. 
Creager. 1995. Effect of nitric oxide synthase inhibition on bleeding time in humans. J. Cardiovasc. Pharmacol. 26:339-342.

18. Bodzenta-Lukaszyke, A., A. Gabryelewicz, A. Lukaszyk, M. Bielawiec, J.W. Konturek, and W. Domschke. 1994. Nitric oxide synthase inhibition and platelet function. Thromb. Res. 75:667-672.

19. Winn, R.K., D. Liggitt, N.B. Vedder, J.C. Paulson, and J.M. Harlan. 1993. Anti-P selectin monoclonal antibody attenuates reperfusion injury to the rabbit ear. J. Clin. Invest. 92:2042-2047.

20. Kestin, A.S., C.R. Valeri, S.F. Khuri, J. Loscalzo, P.A. Ellis, H. MacGregor, V. Birjiniuk, H. Ouimet, B. Pasche, M.J. Nelson, et al. 1993. The platelet function defect of cardiopulmonary bypass. Blood. 82:107-117.

21. Hawiger, J., S. Parkinson, and S. Timmons. 1980. Prostacyclin inhibits mobilization of fibrinogen-binding sites on human ADP- and thrombin-treated platelets. Nature (Lond.). 283:195-198.

22. Born, G.V., and M.J. Cross. 1963. The aggregation of blood platelets. J. Physiol. (Lond.). 168:178-195.

23. Ichimori, K., H. Ishida, M. Fukahori, H. Nakazawa, and E. Murakami. 1994. Practical nitric oxide measurement employing a nitric oxide-selective electrode. Rev. Sci. Instrum. 65:1-5.

24. Holmsen, H., and C.A. Dangelmaier. 1989. Measurement of secretion of serotonin. Methods Enzymol. 169:205-210.

25. Mellion, T.B., L.J. Ignarro, E.H. Ohlstein, E.G. Pontecorvo, A.L. Hyman, and P.J. Kadowitz. 1981. Evidence for the inhibitory role of guanosine 3', $5^{\prime}$-monophosphate in ADP-induced human platelet aggregation in the presence of nitric oxide and related vasodilators. Blood. 57:946-955.

26. Adams, M.R., C.J. Forsyth, W. Jessup, J. Robinson, and D.S. Celermajer. 1995. Oral L-arginine inhibits platelet aggregation but does not enhance endothelium-dependent dilation in healthy young men. J. Am. Coll. Cardiol. 26: 1054-1061.
27. Malinski, T., M.W. Radomski, Z. Taha, and S. Moncada. 1993. Direct electrochemical measurement of nitric oxide released from human platelets. Biochem. Biophys. Res. Commun. 194:960-965.

28. Radomski, M.W., R.M.J. Palmer, and S. Moncada. 1990. An L-arginine/ nitric oxide pathway present in human platelets regulates aggregation. Proc. Natl. Acad. Sci. USA. 87:5193-5197.

29. Valles, J., M.T. Santos, A.J. Marcus, L.B. Safier, M.J. Broekman, N. Islam, H.L. Ullman, and J. Aznar. 1993. Downregulation of human platelet reactivity by neutrophils. Participation of lipoxygenase derivatives and adhesive proteins. J. Clin. Invest. 92:1357-1365.

30. Yao, S.K., J.C. Ober, A. Krishnaswami, J.J. Ferguson, H.V. Anderson, P. Golino, L.M. Buja, and J.T. Willerson. 1992. Endogenous nitric oxide protects against platelet aggregations and cyclic flow variations in stenosis and endothelium injured arteries. Circulation. 86:1302-1309.

31. Celi, A., G. Pellegrini, R. Lorenzet, A. De Blasi, N. Ready, B.C. Furie, and B. Furie. 1994. P-selectin induces the expression of tissue factor on monocytes. Proc. Natl. Acad. Sci. USA. 91:8767-8771.

32. Palabrica, T., R. Lobb, B.C. Furie, M. Aronovitz, C. Benjami, Y-M Hsu, S.A. Sajer, and B. Furie. 1992. Leukocyte accumulation promoting fibrin deposition is mediated in vivo by P-selectin on adherent platelets. Nature (Lond.). 359:848-850.

33. Vita, J.A., C.B. Treasure, E.G. Nabel, J.M. McLenachan, R.D. Fish A.C. Yeung, V.I. Vekshtein, A.P. Selwyn, and P. Ganz. 1990. Coronary vasomotor response to acetylcholine relates to risk factors for coronary artery disease. Circulation. 81:491-497.

34. Freedman, J.E., B. Hankin, C. Alpert, J. Loscalzo, J.F. Keaney, Jr., and J.A. Vita. 1996. Impaired platelet production of nitric oxide in patients with coronary artery disease. Circulation. 94:I-402a (Abstr.). 